Michael Prümer

Cash Flow Management 
Michael Prümer

\section{Cash Flow Management}

Wie Unternehmen langfristig

Liquidität und Rentabilität sichern 
Bibliografische Information Der Deutschen Bibliothek

Die Deutsche Bibliothek verzeichnet diese Publikation in der Deutschen Nationalbibliografie; detaillierte bibliografische Daten sind im Internet über <http://dnb.ddb.de> abrufbar.

1. Auflage 2005

Alle Rechte vorbehalten

(c) Betriebswirtschaftlicher Verlag Dr. Th. Gabler/GWV Fachverlage GmbH, Wiesbaden 2005

Lektorat: Ulrike M. Vetter

Der Gabler Verlag ist ein Unternehmen von Springer Science+Business Media. www.gabler.de

Das Werk einschließlich aller seiner Teile ist urheberrechtlich geschützt. Jede Verwertung außerhalb der engen Grenzen des Urheberrechtsgesetzes ist ohne Zustimmung des Verlags unzulässig und strafbar. Das gilt insbesondere für Vervielfältigungen, Übersetzungen, Mikroverfilmungen und die Einspeicherung und Verarbeitung in elektronischen Systemen.

Die Wiedergabe von Gebrauchsnamen, Handelsnamen, Warenbezeichnungen usw. in diesem Werk berechtigt auch ohne besondere Kennzeichnung nicht zu der Annahme, dass solche Namen im Sinne der Warenzeichen- und Markenschutz-Gesetzgebung als frei zu betrachten wären und daher von jedermann benutzt werden dürften.

Umschlaggestaltung: Nina Faber de.sign, Wiesbaden

Gedruckt auf säurefreiem und chlorfrei gebleichtem Papier

ISBN 978-3-322-96340-6 ISBN 978-3-322-96339-0 (eBook)

DOI 10.1007/978-3-322-96339-0 


\section{Vorwort}

Wenn in einem Unternehmen Cash Flow Management betrieben werden soll, gilt es zunächst zu klären, was überhaupt ein Cash Flow ist. In Lexika, in Wirtschaftszeitungen oder auch von Banken werden unterschiedlichste Definitionen genutzt, um zu beschreiben, was unter Cash Flow zu verstehen ist.

Unabhängig von Definitionen hat Cash Flow zwei Bedeutungsebenen. Zum einen ist der Cash Flow in einer Herkunftsbetrachtung ein Indikator für die Rentabilitätsstärke eines Unternehmens und beantwortet die Frage, welchen Ertrag ein Unternehmen erwirtschaftet. Die zweite Ebene ist die Verwendungsbetrachtung, die der Frage nachgeht, welche liquiden Mittel einem Unternehmen für seine künftige Geschäftstätigkeit zur Verfügung stehen.

Rentabilität und Liquidität sind dabei sehr eng miteinander verbunden. Nur ein Unternehmen, das ausreichend liquide ist, kann überhaupt Geschäfte tätigen. Und wenn diese Geschäfte rentabel sind, wird damit die finanzielle Basis für die Fortführung der Geschäfte aus eigener Kraft gelegt. Zusätzlich wird die Liquidiät allerdings auch durch bilanzielle und andere Maßnahmen beeinflusst.

Es ist nicht Intention dieses Buches, eine neue Definition für Cash Flow den bereits vorhandenen hinzuzufügen. Vielmehr geht es primär darum, eine eher praktische Herleitung des Cash Flows vorzunehmen und die positiven oder negativen Auswirkungen geplanter Maßnahmen sowohl auf die Rentabilität als auch auf die Liquidität darzustellen.

Ein Privatmann kann - simpel betrachtet - nur so viel Geld ausgeben, wie er im Portemonnaie hat. Ähnliches gilt für ein Unternehmen, wobei in diesem Buch an die Stelle des Portemonnaies das Kontokorrentkonto tritt. Viele Faktoren, die den Cash Flow beeinflussen, lassen sich durch Veränderungen des Saldos auf dem Konto darstellen.

Einige andere Faktoren, wie Bewertungsüberlegungen oder Kreditlimite von Banken und Kreditversichern, berühren das Konto nicht. Dennoch haben sie aber über die Rentabilität oder auch außerhalb von Bilanz und Gewinn- und Verlustrechnung Einfluss auf die aktuelle oder zukünftige Liquidität eines Unternehmens

Um diese Faktoren im Einzelnen zu beleuchten, folgt das Buch der Chronologie einer beispielhaft gewählten Unternehmensentwicklung, beginnend mit der Gründungsphase. Häufig ist in der Praxis anzutreffen, dass die für eine Gründung geplanten Mittel nicht ausreichen. Der Gründungsphase folgt dann eine Phase, in der ein entstandener Nachfinanzierungsbedarf gedeckt werden muss.

In den beiden Kapiteln „Gründungsphase“ und „Nachfinanzierungsbedarf“ stehen zwar Finanzierungsfragen, insbesondere über Banken, im Vordergrund. Doch die dafür zu treffenden Vereinbarungen wirken über die Konditionsgestaltung auf die zukünftige 
Vorwort

Rentabilität oder über die Sicherheitenvereinbarungen auf die künftigen Liquiditätsspielräume. Damit haben sie aber einen bedeutenden Einfluss auf das weitere Cash Flow Management eines Unternehmens.

An die beiden ersten Phasen schließt sich die Expansionsphase an, in der die Erweiterung finanzieller Spielräume dargestellt wird. Neben Banken gewinnen nun andere Kreditgeber, wie Kreditversicherer, Leasing- oder Factoringgesellschaften an Bedeutung. Und außer kurz- und mittelfristigen Krediten kommen jetzt ebenso der Diskontund der Avalkredit in Betracht.

Eine Expansionsphase sollte - und so unterstellt es auch das Kapitel - von einer guten oder sehr guten Rentabilität gekennzeichnet sein. Ein hoher Gewinn am Ende des Jahres führt dann zu Bewertungsüberlegungen, die zum einen aus steuerlicher Sicht notwendig sind. Zum anderen dienen sie aber auch dazu, Reserven für die künftige Rentabilität und Liquidität zu legen.

In der Realität ist eine zeitweise negative Entwicklung des wirtschaftlichen Geschehens nichts Ungewöhnliches. Die Zwänge in einer Rezessionsphase stellen an Unternehmer aber hohe Anforderungen. Neben dem Kosten- und Vertriebsmanagement, um das Unternehmen wieder rentabel zu machen, sehen sie sich nun dem zunehmenden Risikodenken der Banken ausgesetzt.

Der Forderung nach zusätzlichen Sicherheiten oder der Gefahr einer Kreditlinienkürzung kann aber das Unternehmen teilweise aus eigener Kraft entgegentreten. Ein aktives Management von Forderungs- und Lagerbeständen wie auch des Anlagevermögens ermöglichen in bestimmten Grenzen eine Liquiditätsschöpfung im Unternehmen, die im günstigen Fall zu einer Rentabilitätsverbesserung führen kann.

Haben Unternehmen die vermeintlich schwierigste Phase der wirtschaftlichen Abschwächung überstanden, stehen sie mit einer beginnenden konjunkturellen Erholung vor den vielleicht größten Anforderungen. Die Abschwungphase hat durch Verluste das Eigenkapital geschwächt. Um die Verluste zu finanzieren, ist auch die Liquidität stark strapaziert worden.

In der Regel fehlt nun die Kraft, aus eigenen Mitteln - sei es durch frisches Kapital von außen oder durch Liquiditätsschöpfung im Unternehmen - die finanzielle Basis zu schaffen, an dem Aufschwung überhaupt teilnehmen zu können. Als Ausweg bleibt nur, mit den Banken die Ausweitung der Kreditlinien vereinbaren zu können.

Natürlich sind die in diesem Kapitel dargestellten Ertrags- und Liquiditätsplanung in jeder Phase der Unternehmensentwicklung für die Gespräche mit Banken erforderlich. Nun sind sie aber unerlässlich, denn sie bilden die einzige noch verbliebene Basis für die weitere Kreditbereitschaft der Banken. Und leider werden sie in mittelständischen Unternehmen immer noch zu wenig genutzt.

Banken wollen aber nicht nur vorab Planungen für die nähere Zukunft erhalten. Ihnen ist auch wichtig, vom Unternehmen über die dann eingetretene Entwicklung informiert zu 
werden. Anhand von Soll-Ist-Vergleichen erwarten sie Begründungen für positive wie vor allem negative Abweichungen gegenüber der Erwartungen.

Die in diesem Kapitel dargestellten Erläuterungen zur wirtschaftlichen Entwicklung des Beispielunternehmens sollen daher auch als Hilfestellung dienen, wenn Banken gegenüber Kommentare zum Soll-Ist-Vergleich anzufertigen sind. Und wie jedes Kapitel schließt auch dieses mit einer Bilanz, einer Gewinn- und Verlustrechnung sowie der Kontokorrententwicklung.

Um die Konsequenzen auf die Entwicklung des Cash Flows deutlich zu machen, ist die dargestellte Unternehmensentwicklung idealtypisch gewählt. Die einzelnen Maßnahmen und ihre Wirkung auf Rentabilität und Liquidität werden so weit möglich unabhängig von den komplexen Geschehnissen im Unternehmensprozess betrachtet.

Insbesondere wird auch auf steuerliche Konsequenzen nicht eingegangen, da für die Beurteilung steuerlicher Wirkungen die Besonderheiten des individuellen Steuerpflichtigen zu betrachten sind, die im Rahmen eines Buches aber nicht berücksichtigt werden können.

Im Buch und insbesondere in den Tabellen werden aus Platzgründen, aber auch um die Lesbarkeit nicht einzuschränken, Geldbeträge in Tausend Währungseinheiten und weitgehend ohne Bezeichnung der Währung dargestellt. 


\section{Inhaltsverzeichnis}

Vorwort

$\begin{array}{ll}\text { Einleitung } & 13\end{array}$

1 Die Gründungsphase $\quad 19$

$\begin{array}{lll}1.1 & \text { Kapitalausstattung } & 20\end{array}$

$\begin{array}{ll}\text { 1.1.1 Bilanzstichtag } & 20\end{array}$

$\begin{array}{ll}1.1 .2 \text { Eigenkapital } & 22\end{array}$

$\begin{array}{lll}\text { 1.1.3 Die Eröffnungsbilanz } & 25\end{array}$

$\begin{array}{lll}1.1 .4 & \text { Fremdkapital } & 25\end{array}$

1.1.4.1 Kurzfristiger Bankkredit - Kreditlinie $\quad 27$

1.1.4.2 Langfristiger Bankkredit 31

$\begin{array}{lll}1.2 & \text { Die Investitionsphase } & 34\end{array}$

1.2.1 Die Bilanz nach Abschluss der Investitionen $\quad 37$

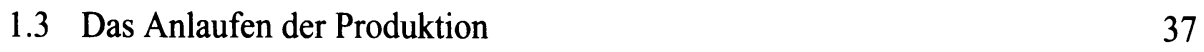

$\begin{array}{ll}\text { 1.3.1 Zahlungsplan } & 38\end{array}$

$\begin{array}{ll}\text { 1.3.2 Zahlungsverhalten } & 40\end{array}$

$\begin{array}{ll}\text { 1.3.3 Personal } & 42\end{array}$

$\begin{array}{lll}\text { 1.3.4 Sonstige Kosten } & 43\end{array}$

$\begin{array}{lll}1.3 .5 & \text { Lieferanten } & 45\end{array}$

1.3.5.1 Zahlungstermine $\quad 45$

$\begin{array}{lll}\text { 1.3.5.2 Zahlungsart } & 46\end{array}$

$\begin{array}{lll}\text { 1.3.5.3 Skonti und Boni } & 48\end{array}$

1.3.6 Die Bilanz vor Abschlussbuchungen $\quad 49$

1.4 Abschluss des Gründungsjahres $\quad 50$

$\begin{array}{lll}\text { 1.4.1 Inventur } & 51\end{array}$

$\begin{array}{ll}\text { 1.4.2 Abschreibungen } & 52\end{array}$

$\begin{array}{lll}1.4 .3 & \text { Rückstellungen } & 53\end{array}$

$\begin{array}{ll}1.4 .4 \text { Wertberichtigungen } & 54\end{array}$

$\begin{array}{ll}\text { 1.4.5 Die erste Jahresbilanz } & 55\end{array}$

2 Nachfinanzierungsbedarf $\quad 59$

2.1 Gründe für den Nachfinanzierungsbedarf 59

$\begin{array}{lll}2.1 .1 & \text { Lieferantenverbindlichkeiten } & 61\end{array}$

$\begin{array}{ll}2.1 .2 & 62\end{array}$

$\begin{array}{lll}2.1 .3 & \text { Anlagevermögen } & 63\end{array}$

2.1.4 Umlaufvermögen $\quad 63$

$\begin{array}{lll}2.1 .5 & \text { Zusammenfassung } & 64\end{array}$

2.2 Das Nachfinanzierungsproblem $\quad 65$ 
$\begin{array}{lll}2.3 & \text { Möglichkeiten der Nachfinanzierung } & 67\end{array}$

2.3.1 Gesellschafterdarlehen 68

2.3.2 Stille Beteiligung und Mezzanine-Kapital 69

2.4 Die zweite Jahresbilanz $\quad 70$

3 Expansionsphase $\quad 73$

3.1 Kreditversicherungen $\quad 73$

3.1.1 Kreditversicherungen ohne Vertragsverhältnis 74

3.1.2 Kreditversicherungen mit Vertragsverhältnis 75

$\begin{array}{lll}3.2 & \text { Leasing } & 77\end{array}$

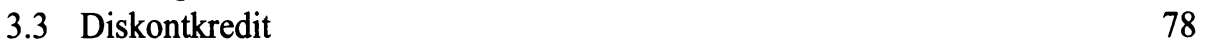

3.3.1 Diskontwechsel von Abnehmern $\quad 79$

$\begin{array}{ll}\text { 3.3.2 Diskontwechsel von Lieferanten } & 80\end{array}$

$\begin{array}{ll}\text { 3.3.3 Scheck-Wechselverfahren } & 81\end{array}$

$\begin{array}{ll}\text { 3.3.4 Zusammenfassung } & 82\end{array}$

3.4 Avalkredit $\quad 82$

3.4.1 Anzahlungsbürgschaften $\quad 83$

3.4.2 Avale von Versicherungsgesellschaften 84

3.5 Die dritte Jahresbilanz vor Bewertungsüberlegungen 85

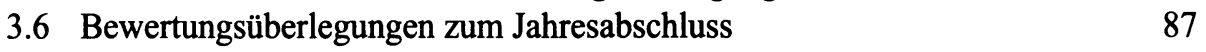

3.7 Die Jahresbilanz nach Bewertungsüberlegungen 88

4 Rezession 91

4.1 Auswirkungen des Umsatzeinbruchs $\quad 92$

4.2 Stabilisierung des Rohertrages 94

4.3 Kostenwirkungen $\quad 96$

4.3.1 Preisbedingter Umsatzrückgang 97

4.3.2 Absatzbedingter Umsatzrückgang 98

4.3.3 Einige Gedanken zu möglichen Maßnahmen 100

4.4 Schwierigere Bankgespräche 103

4.4.1 Steigender Informationsbedarf 104

4.4.2 Zusätzliche Sicherheiten 105

$\begin{array}{ll}\text { 4.4.3 Gleichbehandlungsgrundsatz } & 107\end{array}$

$\begin{array}{lll}\text { 4.4.4 Kreditlinienkürzung } & 109\end{array}$

4.5 Liquiditätsschöpfung im Unternehmen 111

4.5.1 Forderungsmanagement 111

$\begin{array}{ll}\text { 4.5.2 Kreditversicherung } & 113\end{array}$

$\begin{array}{lll}\text { 4.5.3 Factoring } & 113\end{array}$

4.5.4 Forderungen gegenüber Gesellschaftern 114

$\begin{array}{lll}\text { 4.5.5 Lagermanagement } & 115\end{array}$

4.5.6 Management des Anlagevermögens 117

$\begin{array}{ll}\text { 4.6 Die vierte Jahresbilanz } & 119\end{array}$ 
5 Die Konjunkturerholung $\quad 123$

5.1 Ertragsplanung 124

5.2 Die Ertragsplanung des Beispielunternehmens 128

$\begin{array}{ll}5.3 \text { Liquiditätsplanung } & 129\end{array}$

5.4 Die Liquiditätsplanung des Beispielunternehmens 132

5.5 Der Soll-Ist-Vergleich nach sechs Monaten 133

5.6 Der Soll-Ist-Vergleich nach zwölf Monaten 137

$\begin{array}{ll}5.7 \text { Die fünfte Jahresbilanz } & 140\end{array}$

6 Kapitalflussrechnung 145

6.1 Ermittlung des Cash Flows 146

6.2 Cash Flow des Beispielunternehmens über fünf Jahre 149

$\begin{array}{ll}\text { Anhang } & 151\end{array}$

$\begin{array}{ll}\text { Literatur } & 165\end{array}$

$\begin{array}{ll}\text { Stichwortverzeichnis } & 167\end{array}$

$\begin{array}{ll}\text { Der Autor } & 171\end{array}$ 